\title{
Achievements in Reducing the Hospitalization Rate of Home Care Patients in a Regional Hospital in Taiwan
}

\section{Chia-Li H, Yuan-Yi C, Shin-Ping H, Mung-Ting L and Li-Chen S*}

Department of Nursing, Kaohsiung Municipal Ta-Tung Hospital, Kaohsiung Medical University Hospital, Kaohsiung Medical University, Kaohsiung, Taiwan

*Corresponding author: Sun Li-Chen, Department of Nursing, Kaohsiung Municipal TaTung Hospital, Kaohsiung Medical University Hospital, Kaohsiung Medical University, No.68, Jhonghua 3rd Rd, Cianjin District, Kaohsiung City 80145, Taiwan, Tel: (886)0984178796; Email: 730075@ms.kmuh.org.tw

\section{Research Article}

Volume 4 Issue 2

Received Date: April 05, 2021

Published Date: April 22, 2021

DOI: $10.23880 /$ jqhe-16000219

\section{Abstract}

This article provides practical experience of cross-field integration and cooperation in hospitals in a certain region of Taiwan from 2018 to 2019 to reduce the hospitalization rate of home care, reduce the care load of caregivers, improve the quality of care and reduce medical expenses.

Keywords: Hospital; Health Care; Taiwan

\section{Introduction}

Community care is currently actively being promoted to implement long term care in Taiwan.Home health care is an indispensable part of it; besides, it is also long term care under the health insurance payment. Most of the service cases are elderly, multiple chronic diseases cause physical dysfunction, and disease diagnosis.

\section{Purpose}

In order to relieve the loading of caregivers, decrease the proportions of the home health care,raise the quality of the caring, and to reduce the medical expenses.

\section{Method}

This study adopts the research design of medical history retrospective, with descriptive statisticalanalysis. The object of the study was a total of 326 patients who have received care from a homecare center affiliated to a regional hospital from 2018 to 2019 . The data is collected by a structured questionnaire, inclusive of age, gender, discharge disease diagnosis, self-care ability, type of intubation, wound condition, main caregiver at home, number of readmissions, readmission diagnosis, number of days in hospital. After collecting the questionnaire, we workwith physiotherapists, occupational therapists and dietitians to discuss follow-up care plans in cross-disciplinary cooperation, and to teach caregivers related disease care skills, nutrition guidance, passive movement in bed, assist in getting out of bed, and visit the ward during hospitalization to maintain contact with clinical nurses [1,2].

\section{Results}

The average age of the patients in this study was 89 years old. Most of the discharged diseases were diagnosed with pneumonia and heart failure. In the part of self-care ability, the ADL (Barthel Index for Activities of Daily Living) score is 100 points, indicating that the patient cantake care of himself completely. The IADL scale (Lawton And Brody Instrumental Activities ofDaily Living) has a full score of 8 , indicating that the patient can be independent in daily life [3]. 
The highest ADL score of all patients in this study was 24 points. Among them, self-care ability was completely dependent on caregivers, accounting for $94.2 \%$. The highest score in the IADL is 3 , of which $91 \%$ are dependent on caregivers in daily life [4]. The main caregivers are foreign caregivers accounting for $48 \%$, followed by family caregivers accounting for $35 \%$ and domesticcaregivers accounting for $17 \%$. During the hospitalization, the home nurse maintains continuouscontact with the clinical nurse and the personal supervisor, and assists the patient to get out of bed at least twice a day through cross-field care guidance and back smash at least three times.

The caregiver assists the patient to perform upper limb exercises twice a day, raises the head ofthe bed 30 to 45 degrees for the bedridden patient, records the diet and guides food preparation. The hospitalization rate dropped from $10.98 \%$ to $7.7 \%$ from 2018 to 2019.

\section{Conclusion}

Home-care patients are mainly elderly, severely disabled, and intubated. They need long-termcare to maintain their health. The role of the main caregiver at home is very important as well.

They often encounter the problem of caregiver rotation. Insufficient care skills have led to a highrate of hospitalization.
It is recommended to use actual health education guidance, caregivers to respond to the teaching instantly, in addition to guiding foreign care workers or nursing skills, and family members to learn care skills together. Active solicitude during hospitalization is alsovery important to win the trust from patients, as well as continuous contact with clinical nurses and individual supervisors. Care will not be interrupted, and the quality of care is also improvedthrough the study.

\section{References}

1. Hsien HH, Li YC, Ma CC, Lo YK (2012) The Hospitalization Frequency of Nursing Home Residents - AComprehensive Analysis. Taiwan Geriatr Geronto 7(1): 27-40.

2. Lin CJ, Chang YC, Tsou MT, Chen YJ, Hwang LC (2019) Risk Factors Associated with Hospitalization for Pneumonia in Oldest Old Home Care Recipients. Taiwan Journal of Family Medicine 29(1): 34-42.

3. Tai TW, Liu YT, Chen TL (2018) The Reduction of the Incidence Density oflower Respiratory Tract Infection in a Nursing Home. Yuan-Yuan Nursing 12(3): 45-33.

4. Tay WY, Low LL, Tan SY, Vasanwala FF (2014) Evidencebased measures for preventing aspiration pneumonia in patients with dysphagia. Proceedings of Singapore Healthcare 23(2): 45-33. 